\title{
Preoperative NT-proBNP values in patients with atrioventricular septal defect and its role as a predictor of early postoperative course
}

\author{
Bobik L ${ }^{1}$, Kovacikova L ${ }^{1}$, Zahorec $\mathrm{M}^{1}$, Danova $\mathrm{K}^{2}$ \\ Cardiac Intensive Care Unit, Pediatric Cardiac Centre, National Institute of Cardiovascular Diseases, \\ Bratislava, Slovakia. luciabordacova@gmail.com
}

\begin{abstract}
OBJECTIVES: Atrioventricular septal (AVSDC) defect presents by excessive pulmonary blood flow and congestive heart failure in children. This study was designed to identify and compare N-terminal pro-brain natriuretic peptide (NT-proBNP) values in complete and incomplete form at the time of surgery, and evaluation of postoperative course. METHODS: The prospective study included thirty-one patients with complete and fifteen with intermediate forms, undergoing surgery in the period from May 2009 to January 2014. Pre- and post-operative variables were statistically analyzed.

RESULTS: The median value of NT-proBNP was $1420.0 \mathrm{pg} / \mathrm{mL}$, with elevated levels in complete form (1656.0 vs $488.0 \mathrm{pg} / \mathrm{mL}, \mathrm{p}<0.001$ ); age and weight with negative correlation. Other preoperative variables (genetic disorder, atrioventricular valve regurgitation, borderline ventricle) were not significant. Higher incidence of postoperative variables was present in complete form, namely mechanical ventilation $(p<0.03)$, dose and length of vasopressors $(p<0.002)$, ICU stay $(p<0.04)$, and complications $(p<0.05)$. NT-proBNP analysis as a predictor of postoperative course showed an association with longer ventilatory ( $>48$ hours) and inotropic forms of support, ICU stay, and presence of infection.

CONCLUSION: NT-proBNP value might be helpful in the decision-making process of early surgical treatment of complete form of AVSDC, and useful as a predictor of early post-operative course (Tab. 4, Fig. 3, Ref. 14). Text in PDF www.elis.sk.

KEY WORDS: NT-proBNP, atrioventricular septal defect, congestive heart failure, postoperative course.
\end{abstract}

\section{Introduction}

Cardiac biochemical markers - B-type natriuretic peptide (BNP) and N-terminal segment of pro-B-type natriuretic peptide (NT-proBNP) - are widely used as part of cardiologic evaluation in pediatric group of patients $(1,2)$. Long-term exposure to chronic right or left heart overload leads to dilation of heart structures presented by congestive heart failure. Congenital heart defects with a significant left-to-right shunt associated with elevated pulmonaryto-systemic pressure ratio in high pressure lesions are identified to have higher peptide levels (3). Also other authors have shown higher BNP levels in patients with high pressure shunts such as ventricular septal defects compared to low pressure shunts such as atrial septal defect (4). There are data available demonstrating

${ }^{1}$ Cardiac Intensive Care Unit, Pediatric Cardiac Centre, National Institute of Cardiovascular Diseases Bratislava, and ${ }^{2}$ Department of Laboratory Medicine, National Institute of Cardiovascular Diseases, Bratislava, Slovakia.

Address for correspondence: L. Bobik, MD, PhD, Pediatric Cardiac Centre, National Institute of Cardiovascular Disease, Limbova 1, SK-833 01 Bratislava, Slovakia.

Phone: +421.903673190

Acknowledgements: We would like to acknowledge the cooperation of the colleagues in the operating room and biochemical laboratory of our institution. strong correlation of NT-proBNP to right ventricular dilation and pulmonary pressure caused by left-to-right shunts (5). Together with BNP, NT-proBNP is a predictive biomarker in congenital heart disease surgery and considered an independent predictor of prolonged stay in intensive pediatric care (6)

Atrioventricular septal defect represents a congenital heart defect with a combined pathophysiology of ventricular and atrial septal defects, presented by excessive pulmonary blood flow and symptoms of congestive heart failure. This study was designed to identify NT-proBNP values in children with atrioventricular septal defect with congestive heart failure at the time of surgery and to compare complete and incomplete forms of the defect. Subsequently, we evaluated the relationship between these values and postoperative course of patients, such as duration of mechanical ventilation, vasoactive medication, cardiac intensive care unit (ICU) stay and postoperative complications. At the same time we determined the utility of this marker in decision-making process for timing the surgery of these children.

\section{Material and methods}

Study population and demographic data

The prospective observational cohort study included pediatric patients with intermediate and complete forms of atrioventricular 
Tab. 1. Baseline demographic and clinical data.

\begin{tabular}{|c|c|c|}
\hline \multirow[b]{2}{*}{ Data/factor } & \multicolumn{2}{|c|}{ Number of patients $(\mathrm{n}=46)$} \\
\hline & $\begin{array}{c}\text { Complete } \\
\text { form }(\mathrm{n}=31)\end{array}$ & $\begin{array}{l}\text { Intermediate } \\
\text { form }(\mathrm{n}=15)\end{array}$ \\
\hline \multicolumn{3}{|l|}{ Gender } \\
\hline Female & 20 & 7 \\
\hline Male & 11 & 8 \\
\hline \multicolumn{3}{|l|}{ Weight } \\
\hline Mean \pm SD & $4.9 \pm 0.3$ & $7.0 \pm 0.7 *$ \\
\hline Median (range) & $4.8(3.7-13.0)$ & $6.3(2.7-10.2)$ \\
\hline \multicolumn{3}{|l|}{ Age (days) } \\
\hline Mean \pm SD & $185 \pm 23$ & $321 \pm 68$ \\
\hline Median (range) & $161(23-730)$ & $180(115-898)$ \\
\hline Genetic disorder & 24 & 10 \\
\hline Down syndrome & 23 & 9 \\
\hline Down/Turner syndrome & 0 & 1 \\
\hline Preoperative risk factors & 7 & 4 \\
\hline Prematurity & 2 & 1 \\
\hline Hypotonia & 1 & 0 \\
\hline Duodenal atresia/stenosis & 1 & 1 \\
\hline Renal pelvic dilation & 1 & 1 \\
\hline Central nervous system anomaly & 1 & 0 \\
\hline Gastroesophageal reflux disease & 1 & 0 \\
\hline Deafness & 1 & 0 \\
\hline Hypothyroidism & 0 & 2 \\
\hline Associated cardiac lesions & 13 & 7 \\
\hline Right ventricle outflow tract obstruction & 3 & 2 \\
\hline Left ventricle outflow tract obstruction & 0 & 1 \\
\hline Borderline right ventricle & 4 & 1 \\
\hline Borderline left ventricle & 6 & 3 \\
\hline \multicolumn{3}{|l|}{ Preoperative echo cardiac finding } \\
\hline $\begin{array}{l}\text { Right atrioventricular valve orifice (tri- } \\
\text { cuspid) regurgitation mild-moderate }\end{array}$ & 22 & 14 \\
\hline $\begin{array}{l}\text { Left atrioventricular valve orifice (mi- } \\
\text { tral) regurgitation mild-moderate }\end{array}$ & 13 & 10 \\
\hline
\end{tabular}

septal defect, undergoing open-heart surgery at Pediatric Cardiac Centre, The National Institute of Cardiovascular Diseases of Slovakia, Bratislava between May 2009 and January 2014. Informed consent from patient's parents was obtained. During this period, 46 patients met the inclusion criteria while thirty-one consecutive patients were with complete form and fifteen patients with intermediate form who underwent open heart surgery with two-patch technique in complete form, or single pericardial patch for atrium septal defect with suture of ventricular septal component in intermediate form. In both variants, various types of right (tricuspid) and left (mitral) atrio-ventricular valve orifice repair (valvuloplasty) were performed depending on valve anatomy, morphology, and grade of valve regurgitation. Baseline demographic data are shown in Table 1. In 7 patients with complete form, pulmonary artery banding was performed previously to limit the pulmonary blood flow and optimize Qp/Qs ratio. Three other patients with complete form and 2 with intermediate form and pulmonary valve stenosis or right ventricle outflow tract obstruction, required pulmonary valve valvulotomy, resection of outflow tract obstruction, or transannular patch repair. In one patient, subaortic membrane was resected during atrioventricular septal defect repair. In one patient congenital diaphragm defect repair was performed in a single session with cardiac surgery. Fourteen patients with borderline right or left ventricle were included according to morphometric and functional echocardiography criteria (diameter of atrio-ventricular valve orifice, valve area, long axis ventricles ratio, apex forming ventricle, etc.) and this finding was analyzed as a risk factor. Patients with an unbalanced form of the defect with hypoplasia of one ventricle, lesion associated with coarctation of aorta and mitral valve anomaly, also with thyroid gland dysfunction with substitution therapy were excluded. None of the patients had elevated creatinin level and renal function impairment in preoperative period.

The pre-operative variables were recorded in each patient, namely gender, age, weight, genetic disorder, associated extracardiac comorbidities (prematurity, hypotonia, duodenal atresia/stenosis, renal anomalies, central nervous system anomalies, gastro-esophageal reflux disease, deafness and hypothyroidism), associated cardiac lesions (outflow tract obstruction, borderline ventricle with apex forming contralateral ventricle) and pre-operative echocardiography assessment of atrioventricular valve regurgitation (mild to moderate grade of both parts of common atrioventricular valves, namely right - tricuspid and left - mitral types of valve regurgitation). The postsurgical data were analyzed, namely the duration of mechanical ventilation, dose and length of vasoactive drug infusion (dopamine, epinephrine), length of ICU stay, postoperative complications, defined as an infection (respiratory infection, mediastinitis, central line infection, sepsis), effusion detected by ultrasound (fluido/chylothor by ultrasound daily check, ascites), arrhythmia (temporary atrioventricular blockage, atrio-ventricular dyssynchronia, junctional ectopic tachycardia, supraventricular tachycardia), pulmonary hypertension or lung function impairment (the ratio of partial oxygen pressure in arterial blood to the fraction of inspired oxygen $\mathrm{paO}_{2} / \mathrm{fiO}_{2}<300$ without significant residual intracardiac shunt, pulmonary edema, or bilateral infiltrates on chest radiographic appearance).

Blood samples were drawn before the surgery, at the beginning of general anesthesia. NT-proBNP was measured using a commercially available electrochemiluminescence immunoassay $(2 \mathrm{ml}$ syringe with blood sample, Elecsys proBNP, Roche Diagnostics, with dynamic range of $0-35000 \mathrm{pg} / \mathrm{mL}$ ).

Data were analyzed using standard statistical software JMP 5.0.1, SAS. The quantitative data showing normal distribution are presented as mean $\pm \mathrm{SD}$, median and range. NT-proBNP values are expressed in $\mathrm{pg} / \mathrm{mL}$. For two groups of patients $t$-test was used for comparison, one-way analysis (ChiSquare approximation, Wilcoxon/Kruskal-Wallis tests) and bivariate analysis with linear fit model of NT-proBNP by factors was performed. Correlations were performed by Pearson's correlation method. P value of < 0.05 was considered statistically significant.

All procedures performed in studies involving human participants were in accordance with the ethical standards of the institutional research committee (Ethical Committee at National Institute of Cardiovascular Diseases, Slovakia). 


\section{Results}

NT-proBNP analysis and pre/postoperative variables in atrioventricular septal defect forms

Distribution of weight and NT-proBNP values at the time of surgery are demonstrated in Figures 1 and 2. As our results confirmed, the patients with complete form are generally indicated for surgical treatment earlier compared to intermediate form, with median age of 161 vs 180 days, and median weight of 4.8 vs 6.3 $\mathrm{kg}$. Comparing both groups and other characteristics, a genetic disorder, especially Down syndrome, was present in $77.4 \%$ vs 66.7 $\%$, and other preoperative risk factors in $22.5 \%$ vs $26.7 \%$. In the whole group, preoperative NT-proBNP value was with mean of $2419.8 \pm 405.7 \mathrm{pg} / \mathrm{mL}$ (median 1420.0, range: 1602.8 - 3236.8).

Comparing the complete and intermediate forms of the defect, there were significant differences in all postoperative variables (length of mechanical ventilation, ICU stay, dose and length of inotropic support, complications) (Tab. 2). The plasma NT-proBNP levels were elevated in both groups; patients with the complete form with congestive heart failure had significantly higher levels, even with surgery performed at earlier age. In the complete form, postoperative complications such as infection, arrhythmia, ascites and pleural effusion, impaired lung function, pulmonary edema, pulmonary hypertension, and other complications (bleeding, diaphragm paralysis, mediastinal hematoma, thrombus, MV replacement) were present more frequently.

Preoperative variables as risk factors of elevated NT-proBNP values

Univariate analysis showed that preoperative demographic data, namely age and weight are associated with elevated NT-

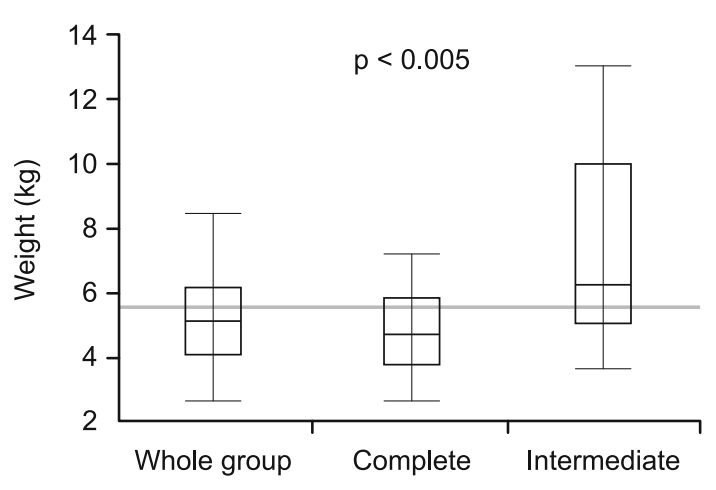

Fig. 1. Box plot of weight distribution by form of atrioventricular septal defect ( 0 - the whole group, 1 - complete form, 2 - intermediate form).

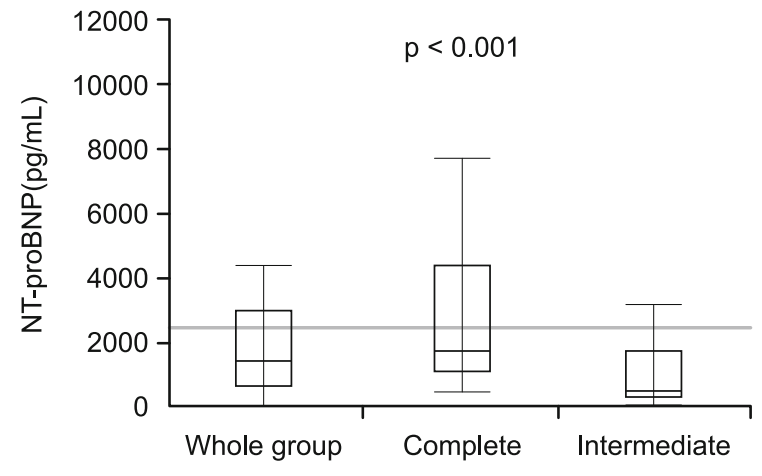

Fig. 2. Box plot of preoperative NT-proBNP distribution by form of atrioventricular septal defect ( 0 - the whole group, 1 - complete form, 2 - intermediate form).

Tab. 2. Preoperative and postoperative variables.

\begin{tabular}{|c|c|c|c|}
\hline Variable & Complete $(n=31)$ & Intermediate $(n=15)$ & $\mathrm{p}$ value \\
\hline \multicolumn{4}{|l|}{ NT-proBNP (pg/mL) } \\
\hline Mean $\pm \mathrm{SD}$ & $3149.7 \pm 547.8$ & $911.0 \pm 228.0$ & $<0.001$ \\
\hline Median (range) & $1656.0(449.0-11754.0)$ & $488.0(87.0-3156.0)$ & \\
\hline \multicolumn{4}{|l|}{ Length of mechanical ventilation (hours) } \\
\hline Mean \pm SD & $53 \pm 11$ & $20 \pm 6$ & $<0.03$ \\
\hline Median (range) & $39(31-240)$ & $13(0-56)$ & \\
\hline \multicolumn{4}{|l|}{ Dose of dopamine } \\
\hline None $/<5 \mathrm{ug} / \mathrm{kg} / \mathrm{min} />5 \mathrm{ug} / \mathrm{kg} / \mathrm{min}$ & $1 / 18 / 12$ & $5 / 9 / 1$ & $<0.003$ \\
\hline \multicolumn{4}{|l|}{ Length of inotropic support (days) } \\
\hline Mean \pm SD & $3.0 \pm 0.4$ & $1.1 \pm 0.3$ & $<0.002$ \\
\hline Median (range) & $2(0-8)$ & $1(0-4)$ & \\
\hline \multicolumn{4}{|l|}{ Length of ICU stay (days) } \\
\hline Mean \pm SD & $5.9 \pm 0.7$ & $3.7 \pm 0.4$ & $<0.04$ \\
\hline Median (range) & $4(2-19)$ & $4(2-7)$ & \\
\hline Infection & 5 & 3 & \\
\hline Ascites, fluidothorax/chylothorax & 3 & 2 & \\
\hline Arrhythmia & 2 & 4 & \\
\hline ALI/ARDS, pulmonary hypertension, edema & 7 & 3 & \\
\hline $\begin{array}{l}\text { Other (bleeding, diaphragm paralysis, mediastinal hematoma, } \\
\text { thrombus, mitral valve replacement) }\end{array}$ & 5 & 0 & \\
\hline
\end{tabular}


Tab. 3. Preoperative variables as a risk factor of elevated NT-proBNP.

\begin{tabular}{|c|c|c|c|}
\hline Factor & Complete $\mathrm{p}$ value & Intermediate $\mathrm{p}$ value & Whole group $\mathrm{p}$ value \\
\hline Age & $<0.02, \mathrm{r}=-0.33$ & $\mathrm{NS}, \mathrm{r}=-0.36$ & $<0.02, \mathrm{r}=-0.34$ \\
\hline Weight & $<0.001, \mathrm{r}=-0.56$ & $\mathrm{NS}, \mathrm{r}=-0.44$ & $<0.0002, \mathrm{r}=-0.52$ \\
\hline Genetic disorder & NS & NS & NS \\
\hline Preoperative atrioventricular valve regurgitation & NS & NS & NS \\
\hline Borderline right/left ventricle & NS & NS & NS \\
\hline
\end{tabular}

Tab. 4. NT-proBNP as a predictor of postoperative course.

\begin{tabular}{|c|c|c|c|}
\hline Factor & Complete & Intermediate & Whole group $\mathrm{p}$ value \\
\hline Mechanical ventilation (hours) & $<0.0008, \mathrm{r}=0.49$ & $<0.04, r=0.56$ & $<0.0002, \mathrm{r}=0.56$ \\
\hline Inotropic agents (days) & $<0.001, \mathrm{r}=0.79$ & $\mathrm{NS}, \mathrm{r}=-0.44$ & $<0.0001, \mathrm{r}=0.80$ \\
\hline ICU stay (days) & $<0.0007, \mathrm{r}=0.47$ & $\mathrm{NS}, \mathrm{r}=0.10$ & $<0.0005, \mathrm{r}=0.52$ \\
\hline Infection & $<0.02$ & NS & $<0.003$ \\
\hline Other complications & NS & NS & NS \\
\hline
\end{tabular}

ICU - intensive care unit

proBNP values with negative correlation (Tab. 3). The presence of genetic syndrome was not associated with elevated NT-proBNP concentration. There was no difference in plasma NT-proBNP concentration in patients with the presence of genetic disorder, preoperative atrioventricular valve regurgitation and right or left borderline ventricle.

\section{NT-proBNP value as a predictor of postoperative course}

The results of NT-proBNP analysis at the time of cardiac surgery as predictors of postoperative course are presented in Table 4. The elevated plasma levels of NT-proBNP were associated with longer ventilatory and inotropic forms of support, longer ICU stay, and the presence of post-operative infection in the whole group of patients. In the subgroup with the complete form, there was a positive correlation between NT-proBNP and post-operative variables. In the subgroup with the intermediate form, NT-proBNP was a predictor of longer duration of mechanical ventilation.

Dividing the patients according to the length of mechanical ventilation did not make any difference in NT-proBNP distribution between early extubated patients (ventilated less than 6 hours) and those ventilated less than 48 hours, in comparison with patients with prolonged ventilator support (Fig. 3).

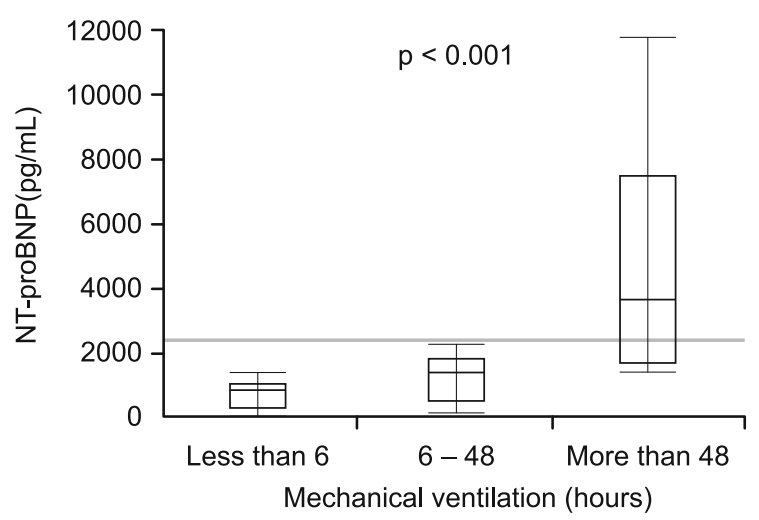

Fig. 3. One-way analysis of NT-proBNP by duration of mechanical ventilation ( $1<6$ hours, $2-6$ to 48 hours, $3>48$ hours).

\section{Discussion}

BNP and NT-proBNP have been studied as biomarkers for the diagnosis, evaluation, and management of heart failure with a high negative predictive value (7). BNP is increased in patients with left-to-right shunt and positively correlates to the shunt volume, systolic right ventricular pressure, mean pulmonary artery pressure, and pulmonary vascular resistance (8). In children, the close correlation between plasma NT-proBNP and modified Ross Score with high sensitivity and specificity can be used as a biochemical marker in the diagnosis and grading of congestive heart failure of patients with ventricular septal defect (9).

Similar to the above mentioned, in children with atrioventricular septal defect, the presence of a large left-to-right shunt leads to an excessive volume loading of both left and right ventricles, followed by hypertrophy and dilatation of ventricles clinically presented by a varying grade of congestive heart failure, and development of pulmonary hypertension. Such clinical course is typical for patients with the complete form of atrioventricular septal defect with early onset of congestive heart failure. The intermediate form presents a subtype of atrioventricular septal defect with the common atrioventricular valve divided into distinct right and left orifices and varying size of inlet ventricular septal defect. Thus this form presents a hemodynamically less serious form with late onset of clinical symptoms. Surgical repair in both forms is performed electively prior to the development of irreversible pulmonary vascular obstructive disease with pulmonary hypertension. Surgical approach (double vs single patch) is performed according to the anatomical finding of atrioventricular septal defect and surgeon's preference. Earlier repair is considered for infants failing to thrive. Definitive early repair of complete atrioventricular septal defect can be performed with excellent results by two-patch technique with complete cleft closure with very low mortality and stable long-term outcomes, even in neonates (10). Thus NT-proBNP may present an additional and helpful marker in the decision-making process for early surgical treatment, especially in younger infants with hemodynamically significant left-to-right shunt.

With respect to the anatomical form of atrioventricular septal defect, we observed that the values of NT-proBNP taken pre- 
surgically were higher in the group with complete compared to intermediate form $(1656 \mathrm{pg} / \mathrm{mL}$ vs $488 \mathrm{pg} / \mathrm{mL})$. This is probably the result of higher secretion of this hormone due to the increased volume and pressure overload in the complete form. While several studies have declared higher concentrations in the newborns and infants $(7,11)$, reference values of NT-proBNP obtained by the same method of measurement in this age range are reported to decline gradually, and after the age of one month are stable. Normal values without gender differences are determined within the age range from 1 month to 1 year with median of $141 \mathrm{pg} / \mathrm{mL}$, and 95th percentile of $646 \mathrm{pg} / \mathrm{mL}$ (3). In our group of patients, there was only one child indicated for surgical treatment in its neonatal period due to severe clinical symptoms of congestive heart failure, with the NT-proBNP value of $7575 \mathrm{pg} / \mathrm{mL}$, thus exceeding the $95^{\text {th }}$ percentile value of normal range.

From the preoperative demographic data, age and weight play a potential role in plasma NT-proBNP concentration. This value increased significantly especially in the complete form of atrioventricular septal defect. Despite younger age and lower weight, the significantly elevated values of NT-proBNP reflect and indicate a hemodynamically more serious form of the defect due to heart failure. According to analysis, the younger age and lower weight at the time of surgical treatment resulted in elevated peptide levels proportionally in the whole group and complete form of AVSD. In the series of Nir et al (3), patients with high-pressure left-to-right shunt (ventricular septal defect, patent ductus arteriosus) had significantly higher peptide levels than patients with low-pressure left-to-right shunt (atrial septal defect). Our result support the findings of Kunii et al (4) of higher BNP levels in patients with ventricular septal defect than in patients with atrial septal defect with comparable pulmonary-to-systemic blood flow ratio (without statistical analysis reported). From other associated lesion, right- or left-sided outflow tract obstruction was also found as a factor of elevated NT-proBNP levels $(11,12)$. In our cohort of patients, the obstruction of outflow tract was present in six of them, and also this anatomical finding may play role in elevated values. Preexisting renal disorder play a role in affecting NTproBNP value; these patients were excluded from the study. Out of pre-operative parameters, serum creatinine level as a marker of renal dysfunction and acute phase protein (C-reactive peptide) were measured in all patients. These have been proved to be the factors influencing the values of NT-proBNP, but in our group of patients they were in normal range preoperatively. Hypothyroidism was present in 2 patients; none required substitution therapy. Patients with thyroid gland dysfunction with substitution treatment were also excluded from the series.

Data collected from post-operative period support the findings of a more serious postoperative course in patients with the complete form in each variable, namely duration of mechanical ventilation, inotropic support, ICU stay, and presence of complications, including postoperative infection. Higher incidence of infections in the complete form may be caused by prolonged ICU stay and other factors mentioned above. In the study of Pérez-Piaya et al (6), preoperative levels of NT-proBNP correlated positively with the length of cardio-pulmonary bypass and length of stay in the criti- cal care unit. According to these authors, the peptide levels may reflect the severity of the lesion, complexity and duration of the surgical procedure with myocardial injury and impaired cardiovascular function, and subsequently to slower recovery. In this work there was a different distribution of patients with congenital heart disease with a heterogeneous spectrum of diagnoses. In our work, we present values in 2 anatomical subtypes of a single diagnosis. The levels of NT-proBNP were significantly higher and are useful as a prognostic factor of post-operative variables, especially in the complete form with hemodynamically significant left-to-right shunt. Our results also support the findings of Esharawy et al (13) and $\mathrm{Wu}$ et al (9), as to the positive correlation of peptide values with the clinical symptoms of congestive heart failure in children with ventricular septal defect and high-pressure left-to-right shunt.

Nonetheless, the predictive value of NT-proBNP in our patients must be considered very carefully, noting the correlation coefficient of mechanical ventilation and ICU stay, even if statistically significant. In our cohort, peptide was detected as a strong predictor of the prolongation of inotropic mechanical forms of support over 48 hours postoperatively. It is surprising, that there was no statistical difference in NT-proBNP values between patients ventilated less than 6 hours, and the interval from 6 to 48 hours (median of 842.0 $\mathrm{pg} / \mathrm{mL}$ vs $1416.0 \mathrm{pg} / \mathrm{mL}$ ), but with significantly elevated values in patients ventilated more than 48 hours (median of $3641,5 \mathrm{pg} / \mathrm{mL}$ ). Only 2 patients with the intermediate form were ventilated more than 48 hours; both with arrhythmia in postoperative course and residual finding of severe mitral valve regurgitation.

Gessler et al (14) provided observations of correlation of preoperative NT-proBNP levels in children scheduled for elective cardiac surgery with the inotropic score and duration of postoperative mechanical ventilation. In their cohort of patients, the inclusion criteria represented infants with congenital heart disease older than 3 months and children with left-to-right shunt were met only in 16 of 40 patients totally. Preoperative NT-proBNP value may serve as a helpful marker during preoperatively follow-up of patients. It can play role in the decision-making process of early surgical treatment taking into account the presentation of heart failure and clinical status. The predictive value of NT-proBNP would be fortified together with the clinical status of patients (heart failure scoring system, age, weight), echocardiography findings and measurements, and other cardiac biomarkers such as troponin, etc.

The main limitations of this study are the small number of patients and absence of correlation with other aspects of congestive heart failure (clinical score system, echocardiographic measurements, analogous cardiac biomarkers).

\section{Conclusion}

Our results of elevated values of NT-proBNP as a result of cardiac overload in patients with atrioventricular septal defect might be helpful in the decision-making process of early surgical treatment, especially in the complete form of the defect. The data suggest that it is a promising marker for monitoring the status of cardiac impairment, risk stratification for open-heart surgery, and can be useful as a predictor of early post-operative course after the 
surgical intervention, especially as to the duration of mechanical ventilation, inotropic support, and ICU stay.

\section{References}

1. Richards A, Cohn J. N-terminal pro-bratin natriuretic peptide. a powerful biomarker of cardiac disease. J Cardiac Failure 2005; 11: S1.

2. Davis G, Bamforth F, Sarpal A, Dicke F, Rabi Y, Lyon ME. B-type natriuretic peptide in pediatrics. Clin Biochem 2006; 39: 600-605.

3. Nir A, Lindinger A, Rauh M et al. NT-proB-type natriuretic Peptide in infants and children: reference values based on combined data from four studies. Pediatr Cardiol 2009; 30: 3-8.

4. Kunii Y, Kamada M, Ohtsuki S et al. Plasma brain natriuretic peptide and the evaluation of volume overload in infants and children with congenital heart disease. Acta Med Okayama 2003; 57: 191-197.

5. Schoen SP, Zimmermann T, Kittner T et al. NT-proBNP correlates with right heart haemodynamic parameters and volumes in patients with atrial septal defects. Eur J Heart Fail 2007; 9: 660-666.

6. Pérez-Piaya MR, Abarca E, Soler V et al. Levels of N-terminal-pro-brain natriuretic peptide in congenital heart disease surgery and its value as a predicitve biomarker. Interact Cardiovasc Thorac Surg 2011; 12: 461- 466.

7. Soldin S, Soldin O, Boyajian A, Taskier MS. Pediatric brain natriuretic peptide and $\mathrm{N}$-terminal pro-brain natriuretic peptide reference intervals. Clin Chim Acta 2006; 366: 304-308.
8. Koch A, Zink S, Singer H. B-type natriuretic peptide in paediatric patients with congenital heart disease. Eur Heart J 2006; 27: 861-866.

9. Wu Y, Chen S, Huang M, Zhang YQ, Sun K, Chen S. N-terminal pro-brain natriuuretic peptide in the diagnosis of congestive heart failure in pediatric patients with ventricular septal defects. World J Pediatr 2006; $1: 40-44$.

10. Bakhtiary F, Takacs J, Cho MY et al. Long-term results after repair of complete atrioventricular septal defect with two-patch technique. Ann Thorac Surg 2010; 89 (4): 1239-1243.

11. Nir A, Bar-Oz B, Perles Z, Brooks R, Korach A, Rein AJ. N-terminal pro-B-type natriuretic peptide: reference plasma levels from birth to adolescence: elevated levels at birth and in heart disease. Acta Paediatr 2004; 93: 603-607.

12. Welisch E, Kleesiek K, Haas N, Norozi K, Rauch R, Filler G. Aminoterminal proB-type natriuretic Peptide levels for monitoring interventions in paediatric cardiac patients with stenotic lesions. Int J Pediat Article 2009; ID 241376, 6 pages.

13. Elsharawy S, Hassan B, Morsy S, Khalifa N. Diagnostic value of $\mathrm{N}$-terminal pro-brain natriuretic peptide levels in pediatric patients with ventriculard septal defect. Egypt Heart J 2012; 64: 241-246.

14. Gessler P, Knirsch W, Schmitt, Rousson V, Von Eckardstein A. $\mathrm{N}$-terminal pro-Brain natriuretic peptide in children with congenital heart defects and open heart surgery. J Pediatr 2006; 148: 372-376.

Received January 15, 2015. Accepted June 26, 2015. 